\title{
Pulsed Radiofrequency as a Minimally Invasive Treatment Option in Anterior Cutaneous Nerve Entrapment Syndrome: A Retrospective Analysis of 26 Patients
}

\author{
Robbert C. Maatman ${ }^{\mathrm{a}, \mathrm{b}}$, e, Monique A.H. Steegers ${ }^{\mathrm{c}}$, Jan-Willem Kallewaard ${ }^{\mathrm{d}}$, \\ Marc R.M. Scheltinga ${ }^{a}$, b, Rudi M.H. Roumen a, b
}

\begin{abstract}
Background: Chronic abdominal pain is occasionally due to entrapped intercostal nerve endings (ACNES, abdominal cutaneous nerve entrapment syndrome). If abdominal wall infiltration using an anesthetic agent is unsuccessful, a neurectomy may be considered. Pulsed radiofrequency (PRF) is a relatively new treatment option for various chronic pain syndromes. Evidence regarding a beneficial effect of this minimally invasive technique in ACNES is lacking. The aim was to assess the effectiveness of PRF treatment in ACNES patients.
\end{abstract}

Methods: A series of ACNES patients undergoing PRF treatment between January 2014 and December 2015 in two hospitals were retrospectively evaluated. Pain was recorded prior to treatment and after 6 weeks using a numerical rating scale (NRS, 0 (no pain) to 10 (worst possible pain)). Successful treatment was defined as $>50 \%$ NRS pain reduction. Patient satisfaction was scored by patient global impression of change (PGIC, $1=$ very much worse, to $7=$ very much improved).

Results: Twenty-six patients were studied (17 women, median age 47 years, range 18 - 67 years). After 6 weeks, mean NRS score had dropped from $6.7 \pm 1.2$ to $3.8 \pm 2.3(\mathrm{P}<0.001)$. A mean $4.9 \pm 1.4$ PGIC score was reported by then. Short term treatment success (6 - 8 weeks) was $50 \%(\mathrm{n}=13,95 \% \mathrm{CI}: 29$ - 71$)$, while $8 \%$ was pain-free on the longer term (median 15 months). Median effect duration was 4 months (range 2 - 26).

Manuscript submitted January 6, 2018, accepted January 23, 2018

aDepartment of Surgery, Maxima Medical Center, Veldhoven, The Netherlands

bSolviMax, Center of Expertise for ACNES and Center of Excellence for Abdominal Wall and Groin Pain, Department of Surgery, Maxima Medical Center, Eindhoven, The Netherlands

'Department of Anaesthesiology, Radboud University Medical Centre, Nijmegen, The Netherlands

dDepartment of Anaesthesiology, Rijnstate Hospital, Velp, The Netherlands ${ }^{\mathrm{e}}$ Corresponding Author: Robbert C. Maatman, Department of Surgery, Maxima Medical Center, PO Box 7777, 5500 MB Veldhoven, The Netherlands. Email: solvimax.resurge@mmc.nl

doi: https://doi.org/10.14740/jocmr3354w
Conclusions: PRF is temporarily effective in half of patients with ACNES. PRF is safe and may be favored in neuropathic pain syndromes as nerve tissue destruction is possibly limited. A randomized controlled trial determining the potential additional role of PRF in the treatment strategy for ACNES is underway.

Keywords: Chronic pain; Abdominal pain; Pulsed radiofrequency; Health burden; Neuropathic pain

\section{Introduction}

In chronic abdominal wall pain (CAWP) syndromes, the abdominal wall harbors a focus responsible for generating and maintaining the pain stimulus. CAWP is often caused by the anterior cutaneous nerve entrapment syndrome (ACNES) [1]. In ACNES, terminal branches of thoracic intercostal nerves are thought to be "entrapped" by a hitherto unidentified event [2]. Patients present with a circumscript pain point within the lateral boundaries of the rectus abdominis muscle. Moreover, skin sensation covering this tender point is altered, and often a positive pinch test and a positive Carnett's test (increased local tenderness by tensing the abdominal muscles) are found $[3,4]$. ACNES incidence rates in an emergency department of a large teaching hospital approximated $2 \%$ in patients presenting with acute abdominal pain [5]. Knowledge on this cause for chronic abdominal wall pain is still limited and it therefore remains a diagnostic challenge for general physicians and specialists $[1$, $6]$.

If patients are diagnosed with this syndrome, a treatment regimen including tender-point injections may be initiated, whereas a neurectomy is only offered to injection therapy refractive patients [7]. This treatment algorithm, based on various randomized controlled trials (RCTs) and large case series, is successful in up to $80-90 \%$ of a random ACNES population [8]. However, less-invasive procedures may also be of potential benefit in patients with ACNES, although solid evidence is currently lacking.

Pulsed radiofrequency (PRF) is an example of such an alternative, minimally invasive treatment. There are two types of radiofrequency $(\mathrm{RF})$ treatment. RF uses a high-frequency alternating current to create a thermal lesion at the targeted nerve 

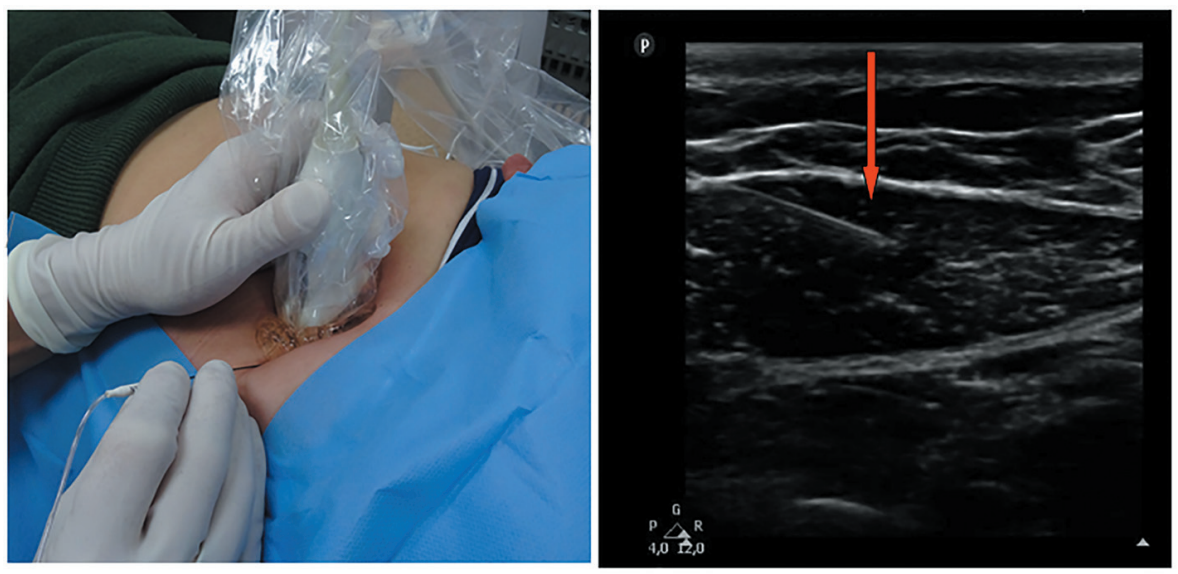

Figure 1. Ultrasound-guided placing of the PRF cannula at the tender point. Right side: the cannula (red arrow) positioned just between the anterior and posterior fascia of the rectus abdominis muscle.

disrupting nociception [9]. Coagulative tissue destruction is accomplished once the probe reaches temperatures up to $80^{\circ} \mathrm{C}$ [10]. RF treatment has been used in various pain syndromes for over 30 years with encouraging results [11-13]. However, RF may also be associated with risks such as deafferentation pain syndromes and neuritis $[10,14,15]$. PRF treatment was designed as a less destructive alternative. PRF uses intermittent administration of high frequency current resulting in tissue temperatures below $42{ }^{\circ} \mathrm{C}$ and therefore, irreversible neuronal damage is prevented $[16,17]$. Several clinical studies demonstrated significantly reduced levels of chronic pain in various pain syndromes using PRF [18-22].

Evidence regarding the potential beneficial use of PRF in ACNES is sparse. Two cases reported on PRF treatment of the dorsal root ganglion (DRG) leading to pain reduction and improvement of quality of life in both $[23,24]$. However, the efficacy of PRF at the level of anterior abdominal wall itself is unknown. Aim of this retrospective case series was to assess the effectiveness of PRF in ACNES patients. Furthermore, unfavorable side effects or complications of PRF treatment were tabulated. If PRF is potentially effective, data may be used for initiating an RCT comparing PRF with a neurectomy in patients with injection recalcitrant ACNES.

\section{Materials and Methods}

\section{General information}

In the past decade, the two senior surgical authors (MS, RR) of Maxima Medical Centre (MMC) in Veldhoven have developed an interest in optimizing the treatment algorithm of patients with ACNES including abdominal injections and operations (neurectomy) [3, 7, 25]. Pain departments of two neighboring hospitals (Rijnstate Hospital, Arnhem (RHA); Radboud University Medical Center, Nijmegen, UMCN) have experience in using PRF at the rectus abdominis muscle level in patients with ACNES. Patient charts were retrospectively reviewed by the first author. Local ethics committees of the two participating hospitals approved the study protocol and decided the Medical Research Involving Human Subjects Act (in Dutch: WMO did not apply to). The present analysis was considered auditing of own results and evaluation of patient-reported outcomes.

\section{Eligibility criteria}

Patients with ACNES as determined by pain specialists of RHA and UMCN were eligible for the study if they were $>18$ years old, if they experienced locoregional abdominal pain for at least 1 month, and if they met the following criteria associated with ACNES [7]: 1) Unilateral constant area of tenderness located in the abdominal area with a small area (a few square $\mathrm{cm}^{2}$ ) of maximal intensity situated within the lateral boundaries of the rectus abdominis muscle. 2) Tenderness was aggravated by abdominal muscle tensing using the Carnett test. 3) A larger area of altered skin sensation such as hypoesthesia, hyperesthesia or altered cool perception covering this maximal pain point, but not necessarily corresponding to a specific complete dermatome. 4) Absence of laboratory or imaging abnormalities. Patients were excluded if they had undergone earlier PRF treatment at the dorsal root ganglion (DRG) or had received previous surgical treatment for ACNES. Furthermore, cognitive impairment, recent intra-abdominal pathology, other chronic pain syndromes or spinal surgical procedure at or between vertebral levels T7-L1 were also considered exclusion criteria.

\section{PRF procedure}

Pain specialists of each of the two participating hospitals all had $>5$ years of experience in using PRF. Once a patient is diagnosed with ACNES, he/she is counselled on the specifics of the PRF. If verbal consent is obtained, the patient is placed supine on a stretcher. The patient is asked to locate the point of maximal pain. This often small area is marked with a pencil and the overlying skin is prepped with chlorhexidine ${ }^{\circledR}$ and draped. Guided ultrasound will then be applied in order 


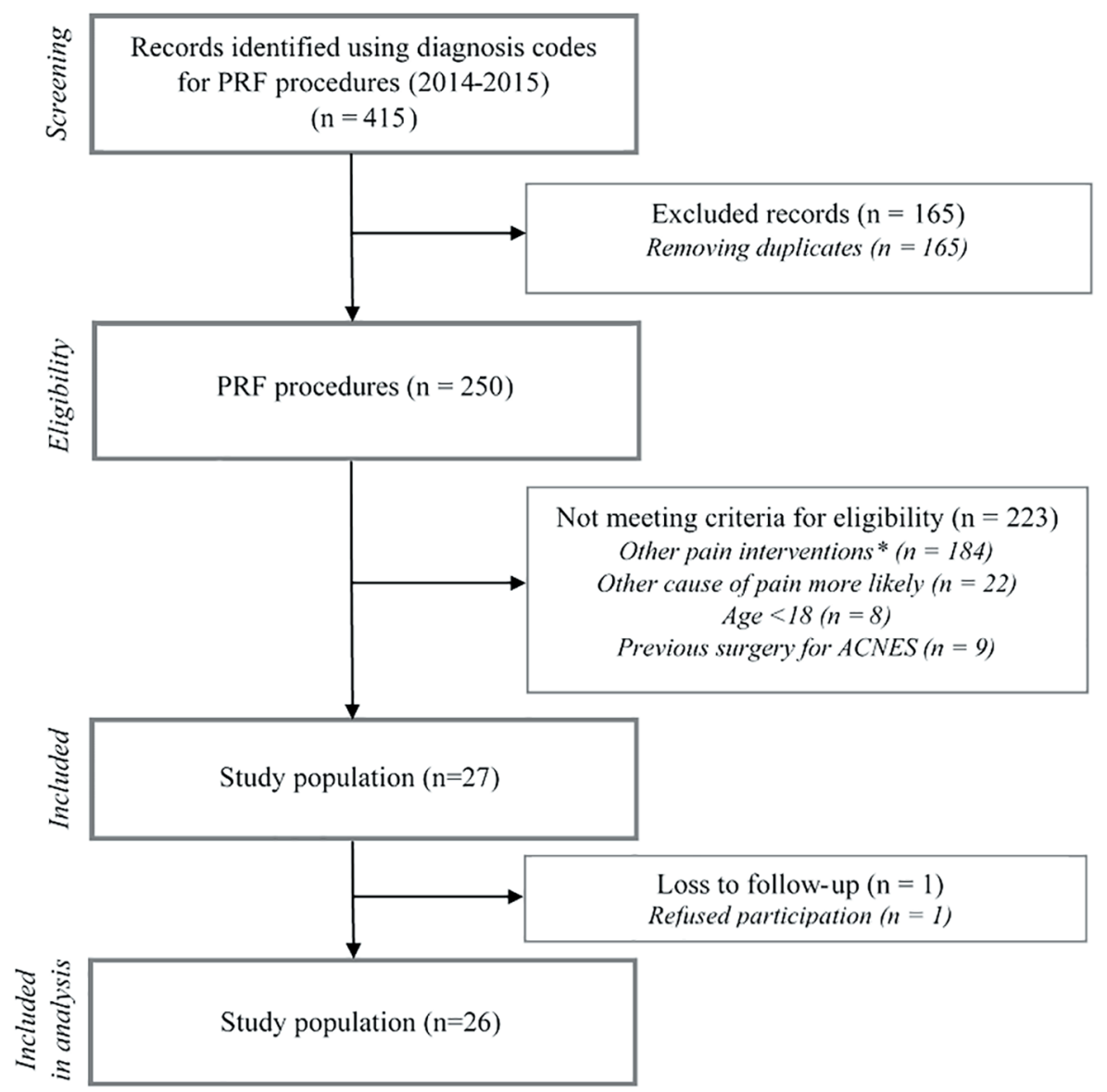

Figure 2. Study flow chart. Other pain interventions included PRF therapy used at the level of the DRG, cryoneuroablation therapy, ultrasound guided trigger-point infiltrations or iontophoresis therapy.

to locate the underlying fascia of the rectus abdominis, which should be located around $2-3 \mathrm{~cm}$ lateral from the linea alba. Once the location is identified, the skin will be anesthetized with $1 \%$ lidocaine.

A straight, sharp RF cannula (SMK Pole $54 \mathrm{~mm}$ needle with $5 \mathrm{~mm}$ active tip, Cotop International BV, Amsterdam, The Netherlands) is used that is connected to a PRF Generator (G4, Cosman Medical, Burlington, MA at the UMCN site; NeuroTherm NT1100 machine, Middleton, MA, at the RHA site) and inserted at a $45^{\circ}$ angle through the skin and its tip is positioned between the anterior and posterior fascia of the rectus abdominis muscle (Fig. 1). A closed electrical circuit stimulation is maintained at a $50 \mathrm{~Hz}$ frequency to obtain a sensory stimulation threshold. Tingling sensations occurred at less than $0.5 \mathrm{~V}$ in all patients and are considered crucial in determining location of the affected nerve [26]. PRF treatment is then applied for 6 min using settings: $45 \mathrm{~V},<42{ }^{\circ} \mathrm{C}, 20 \mathrm{~ms}, 2$ PPS, impedance $<500 \mathrm{Ohm}$, while maintaining the cannula in the very same spot.

On a regular basis after completing PRF therapy, a local anesthetic agent such as ropivacaine $1 \mathrm{~mL} 0.2 \%$ combined with $40 \mathrm{mg}$ of methylprednisolone is left into the area of treatment on indication. On indication was defined as patients having discomfort when their recognizable pain was elicited dur- ing treatment. Patients were allowed to treat residual pain after PRF using analgesic medication as before.

\section{Patient evaluation and data accrual}

A search using specific treatment codes associated with PRF was performed in both participating hospitals, aimed at identifying patients who had received PRF treatment for ACNES. If a hospital was not using electronic data registration, written data logs were manually searched. If certain aspects of indication or treatment were unclear, the responsible doctor was asked for clarification.

Characteristics such as history, age, sex, body height, weight and pain-related specifics of eligible patients receiving PRF between January 2014 and December 2015 were entered in a separate anonymized database. Pain levels and patient satisfaction were measured before and approximately $6-8$ weeks after PRF, according to local treatment protocols. Pain was scored as the average pain level that was experienced at the follow-up visit, using a numerical rating scale (NRS, 0 (no pain) to 10 (worst possible pain)). Patient satisfaction was recorded using the patient global improvement of change (PGIC) scale. PGIC is a 7-point scale depicting a patient's rating of 
Table 1. Characteristics of Patients Receiving PRF for ACNES

\begin{tabular}{|c|c|}
\hline & ACNES patients $(n=26)$ \\
\hline Age (range) & $47(18-67)$ \\
\hline Gender, F/M ratio & $17: 9$ \\
\hline BMI, $\mathrm{kg} / \mathrm{m}^{2}$ & $25.3(17.5-48.4)$ \\
\hline Diagnostic delay, months & $23(2-216)$ \\
\hline \multicolumn{2}{|l|}{ Etiology (n) } \\
\hline Spontaneous & 15 \\
\hline Sport/trauma & 1 \\
\hline After flu/infection & 1 \\
\hline Previous abdominal surgery & 9 \\
\hline Pain (NRS, 0 - 10) & $6.7(1.2)$ \\
\hline \multicolumn{2}{|l|}{ Abdominal pain location, $\mathrm{n}$} \\
\hline Right lower quadrant & 10 \\
\hline Right upper quadrant & 6 \\
\hline Left lower quadrant & 8 \\
\hline Left upper quadrant & 2 \\
\hline
\end{tabular}

Data are presented as means with standard deviation $( \pm S D)$ or median values (range). BMI: body mass index.

overall improvement. Patients judged the current condition as "very much worse" $=1$ to "very much improved" = 7 [27]. PGIC scores of 6 or 7 were considered as a positive outcome. Patient telephone interviews between February and June, 2016 included questions on present levels of pain, satisfaction and long-term complications. A successful outcome was defined as $>50 \%$ pain relief (as calculated using the NRS-scale) and a PGIC score of $>5$.

\section{Data analysis}

All analyses were performed using the Statistical Package for the Social Sciences (SPSS) version 21 for Windows. Categorical variables were described as frequencies. Continuous data were tested for normality and are presented as means with standard deviation $( \pm$ SD) or median values (interquartile range, IQR) as appropriate. Changes in pain scores after or PRF treatment were evaluated using the Wilcoxon signed rank test. Subanalysis was done to compare between treatment with and without the use of corticosteroids. A P-value of $<0.05$ was considered significant.

\section{Results}

\section{Baseline characteristics}

Using our defined search strategy, a total of 415 PRF treatment records were identified in both participating hospitals between January 2014 and December 2015 (Fig. 2). After removing duplicates, 250 unique patients were assessed for eligibility. A total of 223 patients were excluded for reasons as depicted (Fig. 2). Therefore, 27 patients with ACNES fulfilled all study criteria. One patient was lost to follow-up leading to a 26 patient study population. Prior to PRF, all of these 26 patients had received a (temporarily) successful diagnostic injection ( $\geq$ $50 \%$ pain reduction) followed by one or more repetitive analgesic injections. However, this strategy did not result in sufficient long term relief. Baseline characteristics are depicted in Table 1. Median age was 48 years (range 18 - 80). Diagnostic delay prior to the diagnosis of ACNES was 16.5 months (range 2 - 216).

\section{Short term results $(n=26,6$ weeks post PRF $)$}

A mean $6.7 \pm 1.2(95 \%$ CI: 6.2 - 7.1) NRS score was recorded before PRF treatment. Six weeks later, mean NRS scores had dropped to $3.8 \pm 2.3$ (95\% CI: $2.9-4.8)$; $\mathrm{P}<0.001$. At this point, 13 of 26 patients $(50 \%$; 95\% CI: 29 - 71) considered their outcome as successful (defined as $\geq 50 \%$ pain reduction on NRS scale). All 13 patients reported a PGIC of $>5$. A mean $4.9 \pm 1.4$ PGIC score was identified in the whole study population. The duration of pain before diagnosis did not differ between successful responders and failures (median 24 months, range 6 - 216 vs. median 18 months, range 3 - 48; $\mathrm{P}=0.53$ ). No neurological complications or side effects were determined. A subgroup analysis allowed determining whether use of corticosteroids conferred any treatment effect. Mean differences in pain scores and patient satisfaction after PRF treatment are shown in Table 2. A total of 13 patients received corticosteroids after treatment whereas 13 did not for unknown reasons, but most probably because of doctors' preference. In this subgroup analysis, changes in NRS scores as well as patient satisfaction were not statistically different among groups (Table 2).

\section{Long-term follow-up of $(n=26,15$ months $)$}

Long term outcome is depicted in Figure 3 (median, 15 months

Table 2. Subgroup Analysis Corticosteroids Versus No Corticosteroids

\begin{tabular}{lllll}
\hline & Corticosteroids $(\mathbf{n}=\mathbf{1 3})$ & No use of corticosteroids $(\mathbf{n}=\mathbf{1 3})$ & Mean difference $(\mathbf{9 5} \% \mathbf{C I})$ & P-value \\
\hline Pain pre treatment (NRS, 0 - 10) & $6.5 \pm 1.3$ & $6.9 \pm 1.0$ & $0.4(-0.5$ to 1.4$)$ & 0.37 \\
Pain post treatment (NRS, 0 - 10) & $3.6 \pm 1.5$ & $4.1 \pm 2.9$ & $0.5(-1.4$ to 2.3$)$ & 0.61 \\
Mean change & $2.8 \pm 2.0$ & $2.8 \pm 2.2$ & $0.0(-1.7$ to 1.7$)$ & 0.96 \\
Patient satisfaction (PGIC, 0 - 7) & $5.1 \pm 1.3$ & $4.7 \pm 1.5$ & $-0.4(-1.5$ to 0.7$)$ & 0.48 \\
\hline
\end{tabular}

Data are presented as means with standard deviation $( \pm S D)$. 


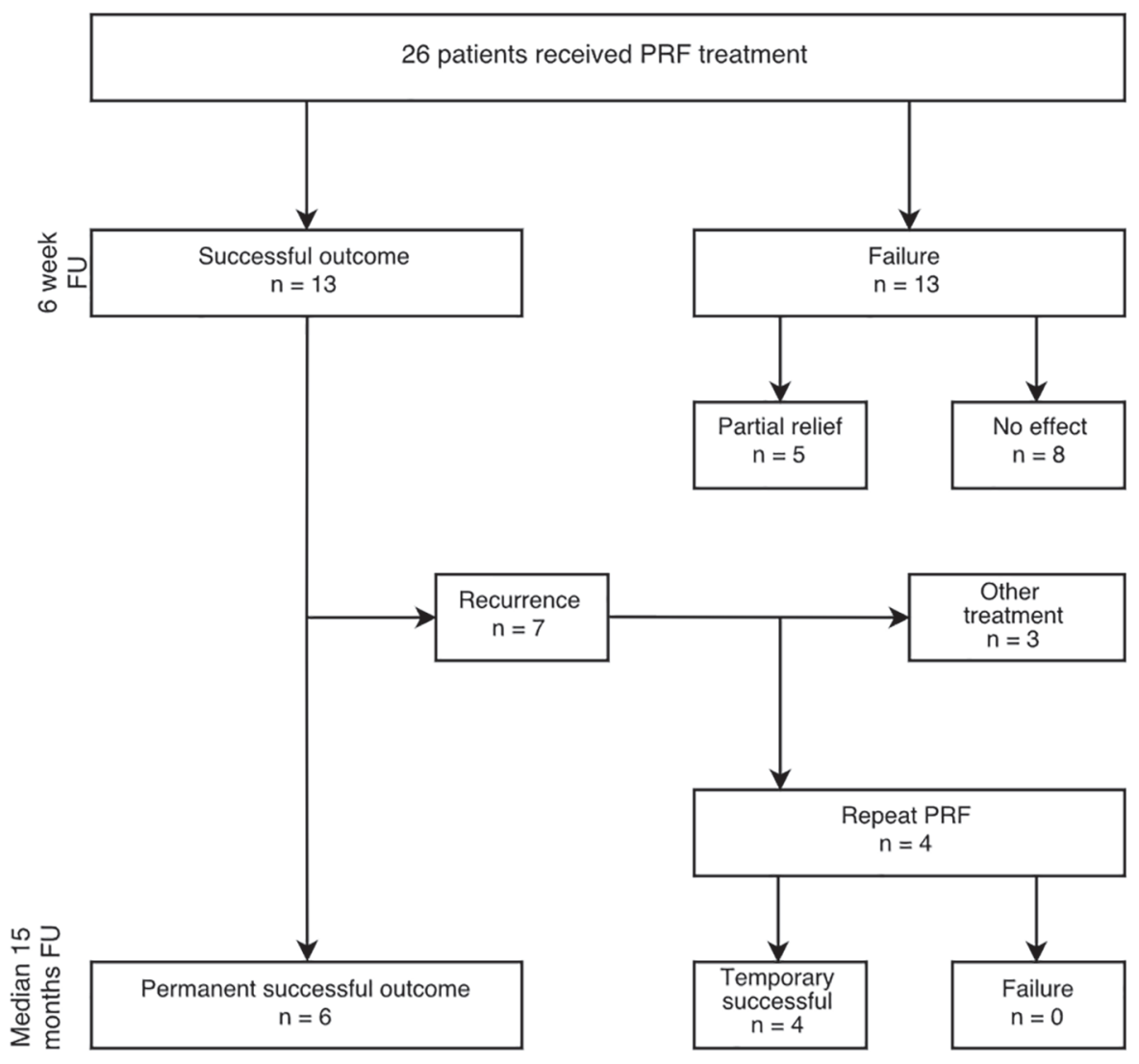

Figure 3. Outcome after PRF treatment in patients with ACNES insufficiently responding to abdominal wall injections. Successful outcome is defined as $>50 \%$ reduction of pain during $>6$ weeks; temporary successful outcome is defined as $>50 \%$ pain reduction for $>6$ weeks before repeating the procedure; long-term follow-up (FU) is median 15 months (range 3 - 26).

(range 3 - 26, 100\% response rate). At that time point, six patients reported success (Fig. 3). Of these six successful patients $(23 \%)$, two were pain free on the long term. PRF treatment was effective for a median 4 months (IQR 3 - 22) (Kaplan-Meier curve, Fig. 4). A steep loss of analgesic effect was observed anywhere between 2 and 5 months after treatment. Four patients underwent a second procedure resulting in success in all. This pain relief was sustained for approximately 3 - 4 months before its effect again wore off.

\section{Discussion}

Several studies in ACNES have indicated that surgery such as a neurectomy at the level of the anterior rectus sheath is effective in the majority of patients $[7,8]$. However, less invasive treatment options may also be beneficial as suggested by two case reports $[23,24]$. The aim of the present retrospective case series was to address the efficacy and current practice of peripheral PRF therapy in ACNES patients who only temporarily responded positive to abdominal wall infiltrations. The results of the present study demonstrate that PRF provides success (defined as a $>50 \%$ drop in pain score) in half of the patient population whereas neurological deficits or other adverse side effects were absent. After 1 year, a quarter of ACNES patients reported an ongoing success, whereas $8 \%$ (two out of 26 ) were still totally pain free. Therefore, PRF therapy appears of potential benefit as a less invasive alternative to surgical intervention in a small portion of patients with ACNES.

Most researchers would argue that minimally invasive techniques such as PRF (and RF) are mainly considered for short term relief [28]. One review demonstrated that a therapeutic effect of PRF is seldom detected beyond 6 months [21]. The largest controlled trial evaluating PRF for occipital neuralgia found that potential beneficial effects lasted for over 6 months [29]. Interestingly, repeating PRF treatment may also be explored although pain relief is not necessarily longer after each session [30-32]. The present retrospective data set in a population with ACNES patients suggests that PRF has an identical short term success rate. A Kaplan-Meier analysis illustrates that a beneficial effect diminishes beyond the 5 month time point in most patients. Interestingly, untoward side effects of PRF were never reported, even after repetitive administration.

PRF (and RF) treatment are practiced on a large scale for several pain conditions in Dutch pain centers. Moreover, PRF was also used for subgroups of ACNES patients, although high level evidence for this indication is currently absent. We have no clue on what scale this type of treatment is utilized for ACNES in other countries. Evidence favoring PRF treatment in 


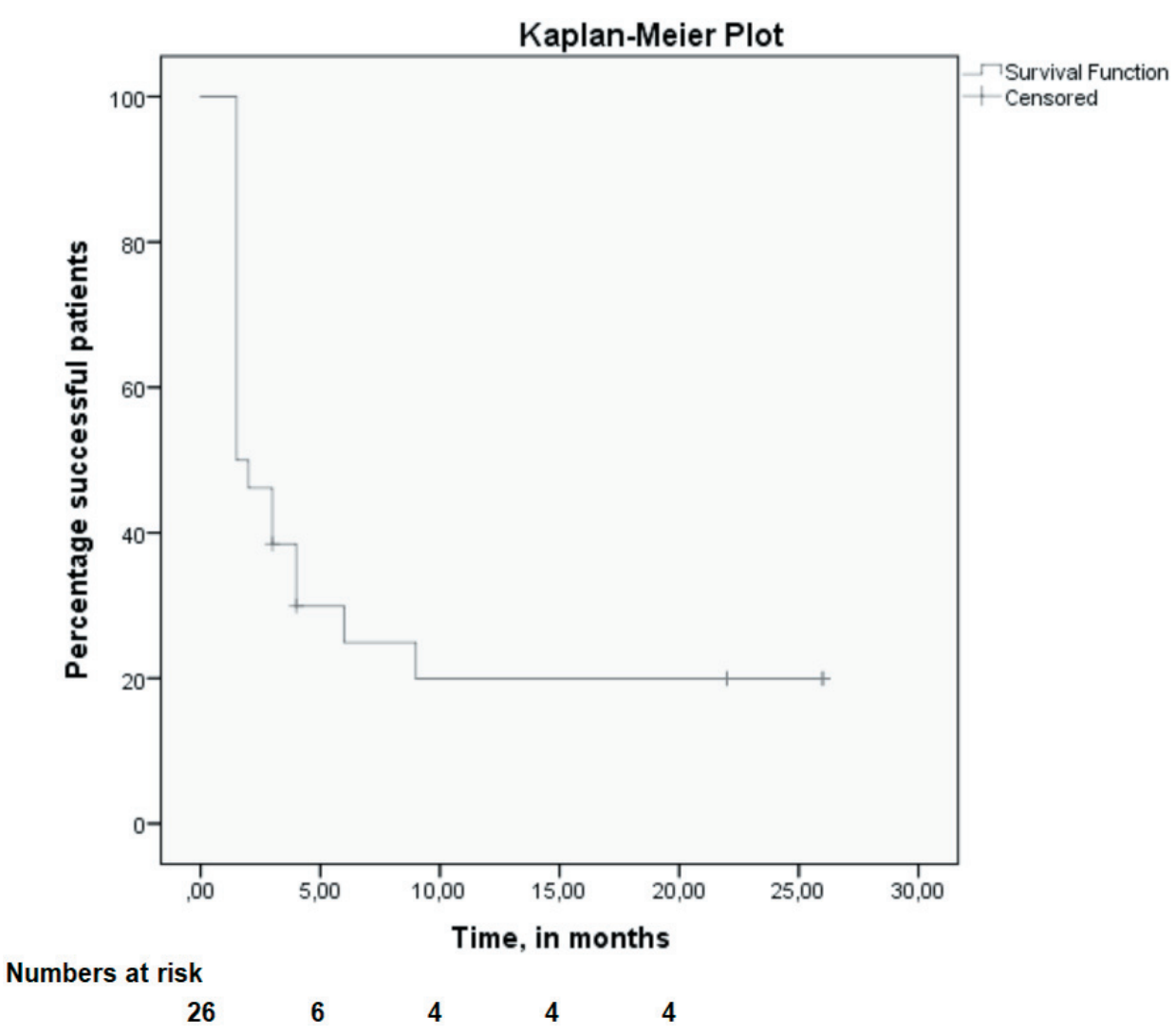

Figure 4. Kaplan-Meier plot. Time dependent success (> 50\% pain reduction, Y-axis) of treatment is presented using the KaplanMeier method, illustrated as a survival curve.

ACNES patients is scarce and limited to two case reports [23, 24]. A similar lack of high level data supporting the use of PRF in other chronic pain conditions is observed [33-35]. Since there is no standard treatment protocol and little evidence regarding the use of PRF in ACNES patients, its efficacy should be established with a well-designed RCT.

ACNES is hypothesized to be a neuropathic pain syndrome. Neuropathic pain has been defined by the International Association for the Study of Pain (IASP) as pain caused by a (demonstrable) lesion or disease of the somatosensory nervous system [36]. Due to the retrospective nature of this study, the presence of neuropathic pain was not demonstrated by objective tests evaluating somatosensory disturbances such as laser evoked potentials, nerve biopsies, quantitative sensory testing or MRI [37]. We also did not standardly use specific questionnaires focusing on neuropathic pain including DN-4, pain DETECT or the neuropathic pain syndrome inventory [37]. However, the mechanical theory proposed by Applegate suggests that terminal parts of cutaneous intercostal nerve branches are "entrapped" in the rectus abdominis muscle and thus leading to an "entrapment neuropathy" [4]. This theory is strengthened by our observations following a neurectomy procedure, in which these terminal branches are ligated and removed, leading to the disappearance of pain and somatosensory disturbances [3]. Therefore, we are confident that the nature of pain as observed in ACNES is neuropathic.

A remarkable observation in our study was the fact that both participating hospitals regularly administered corticosteroids after PRF. The local administration of these agents may possibly have influenced treatment results, but its role on pain reduction is not clearly understood. A clinically relevant effect of steroids was absent in the present study. It remains debatable whether pain relief is caused by the use of steroids, since four out of the six successful patients at long-term follow-up never received steroid treatment. In addition, we recently finished a trial in 136 new and previously untreated ACNES patients evaluating the role of adding corticosteroids to abdominal wall tender point lidocaine injections. Interestingly, no beneficial effect of corticosteroids was found.

The present study harbors flaws including its retrospective character whereas a relatively small sample size limits its power and generalizability. Furthermore, a control group eliminating the bias of the placebo effect of a novel treatment option or spontaneous resolution of symptoms is lacking. A previous study showed that a placebo effect of invasive procedures is possibly higher compared to oral medication pills [38]. In addition, some authors suggested that improvement of symptoms that is not obtained in a blind manner is per definition caused by a placebo effect. Therefore, a potential placebo effect cannot be ruled out. However, the results of our study on PRF in ACNES patients must be seen in the proper context. It must be appreciated that most of our patients were referred after a median of over half a year of diagnostic delay suggesting that this pain syndrome is still frequently overlooked as a cause of 
chronic abdominal wall pain $[1,39]$. It is thus unlikely that the beneficial effect of PRF can be seen solely as placebo effect, since other therapies failed to achieve any success. In addition, a randomized sham-controlled trial showed a beneficial effect for PRF against sham intervention [19]. It must be realized that this study represents the only case series on PRF in ACNES at present providing insight on a "proof of principle" regarding the efficacy of PRF. However, an RCT that is currently underway will identify a possible placebo effect.

In conclusion, the present case series is the first to indicate that PRF is a minimally invasive treatment option that may attain a role in the step up treatment algorithm of patients with ACNES. PRF may be favored in neuropathic pain syndromes as nerve tissue destruction is possibly limited whereas side effects are absent. The use of corticosteroids seems to have no additional beneficial value. An RCT is warranted to clarify the possible role of PRF and is currently underway at our institute (Netherlands Trial Registration: NTR5131) in order to provide level $1 \mathrm{~b}$ evidence for the use of PRF in ACNES patients.

\section{Financial Disclosure}

None.

\section{Conflict of Interest}

None.

\section{References}

1. Srinivasan R, Greenbaum DS. Chronic abdominal wall pain: a frequently overlooked problem. Practical approach to diagnosis and management. Am J Gastroenterol. 2002;97(4):824-830.

2. Applegate WV, Buckwalter NR. Microanatomy of the structures contributing to abdominal cutaneous nerve entrapment syndrome. J Am Board Fam Pract. 1997;10(5):329-332.

3. Boelens OB, van Assen T, Houterman S, Scheltinga MR, Roumen RM. A double-blind, randomized, controlled trial on surgery for chronic abdominal pain due to anterior cutaneous nerve entrapment syndrome. Ann Surg. 2013;257(5):845-849.

4. Applegate W. Abdominal Cutaneous Nerve Entrapment Syndrome (ACNES): a commonly overlooked cause of abdominal pain. Perm J. 2002;6:20-27.

5. van Assen T, Brouns JA, Scheltinga MR, Roumen RM. Incidence of abdominal pain due to the anterior cutaneous nerve entrapment syndrome in an emergency department. Scand J Trauma Resusc Emerg Med. 2015;23:19.

6. Hershfield NB. The abdominal wall. A frequently overlooked source of abdominal pain. J Clin Gastroenterol. 1992;14(3):199-202.

7. Boelens OB, Scheltinga MR, Houterman S, Roumen RM. Management of anterior cutaneous nerve entrap- ment syndrome in a cohort of 139 patients. Ann Surg. 2011;254(6):1054-1058.

8. van Assen T, Boelens OB, van Eerten PV, Scheltinga MR, Roumen RM. Surgical options after a failed neurectomy in anterior cutaneous nerve entrapment syndrome. World J Surg. 2014;38(12):3105-3111.

9. Uematsu S, Udvarhelyi GB, Benson DW, Siebens AA. Percutaneous radiofrequency rhizotomy. Surg Neurol. 1974;2(5):319-325.

10. Byrd D, Mackey S. Pulsed radiofrequency for chronic pain. Curr Pain Headache Rep. 2008;12(1):37-41.

11. van Kleef $M$, Barendse GA, Dingemans WA, Wingen C, Lousberg R, de Lange S, Sluijter ME. Effects of producing a radiofrequency lesion adjacent to the dorsal root ganglion in patients with thoracic segmental pain. Clin J Pain. 1995;11(4):325-332.

12. Lord SM, Barnsley L, Wallis BJ, McDonald GJ, Bogduk N. Percutaneous radio-frequency neurotomy for chronic cervical zygapophyseal-joint pain. N Engl J Med. 1996;335(23):1721-1726.

13. Tekin I, Mirzai H, Ok G, Erbuyun K, Vatansever D. A comparison of conventional and pulsed radiofrequency denervation in the treatment of chronic facet joint pain. Clin J Pain. 2007;23(6):524-529.

14. McDonald GJ, Lord SM, Bogduk N. Long-term followup of patients treated with cervical radiofrequency neurotomy for chronic neck pain. Neurosurgery. 1999;45(1):6167; discussion 67-68.

15. Abbott Z, Smuck M, Haig A, Sagher O. Irreversible spinal nerve injury from dorsal ramus radiofrequency neurotomy: a case report. Arch Phys Med Rehabil. 2007;88(10):1350-1352.

16. Sluijter ME, Cosman ER, Rittman WB, Van Kleef M. The effects of pulsed radiofrequency fields applied to the dorsal ganglia - a preliminary report. Pain Clin. 1998;11:109117.

17. Ahadian FM. Pulsed radiofrequency neurotomy: advances in pain medicine. Curr Pain Headache Rep. 2004;8(1):3440.

18. Philip CN, Candido KD, Joseph NJ, Crystal GJ. Successful treatment of meralgia paresthetica with pulsed radiofrequency of the lateral femoral cutaneous nerve. Pain Physician. 2009;12(5):881-885.

19. Van Zundert J, Patijn J, Kessels A, Lame I, van Suijlekom $H$, van Kleef $M$. Pulsed radiofrequency adjacent to the cervical dorsal root ganglion in chronic cervical radicular pain: a double blind sham controlled randomized clinical trial. Pain. 2007;127(1-2):173-182.

20. Van Boxem K, de Meij N, Kessels A, Van Kleef M, Van Zundert J. Pulsed radiofrequency for chronic intractable lumbosacral radicular pain: a six-month cohort study. Pain Med. 2015;16(6):1155-1162.

21. Chua NH, Vissers KC, Sluijter ME. Pulsed radiofrequency treatment in interventional pain management: mechanisms and potential indications-a review. Acta Neurochir (Wien). 2011;153(4):763-771.

22. van Boxem K, van Eerd M, Brinkhuizen T, Patijn J, van Kleef M, van Zundert J. Radiofrequency and pulsed radiofrequency treatment of chronic pain syndromes: the 
available evidence. Pain Pract. 2008;8(5):385-393.

23. Luis Tellez Villajos BHO, Victor Moreira Vicente, Jose Luis de la Calle Reviriego, Carla Senosiain Lalastra, Jose Ramon Foruny Olcina. Radiofrecuencia pulsada en el tratamiento del sindrome de atrapamiento del nervio cutaneo abdominal. Gastroenterologia y Hepatologia. 2015;38:14-16.

24. Birthi P, Calhoun D, Grider JS. Pulsed radiofrequency for chronic abdominal pain. Pain Physician. 2013;16(4):E443-445.

25. Boelens OB, Scheltinga MR, Houterman S, Roumen RM. Randomized clinical trial of trigger point infiltration with lidocaine to diagnose anterior cutaneous nerve entrapment syndrome. Br J Surg. 2013;100(2):217-221.

26. Ford DJ, Pither C, Raj PP. Comparison of insulated and uninsulated needles for locating peripheral nerves with a peripheral nerve stimulator. Anesth Analg. 1984;63(10):925-928.

27. Dworkin RH, Turk DC, McDermott MP, Peirce-Sandner S, Burke LB, Cowan P, Farrar JT, et al. Interpreting the clinical importance of group differences in chronic pain clinical trials: IMMPACT recommendations. Pain. 2009;146(3):238-244.

28. Mikeladze G, Espinal R, Finnegan R, Routon J, Martin D. Pulsed radiofrequency application in treatment of chronic zygapophyseal joint pain. Spine J. 2003;3(5):360-362.

29. Cohen SP, Peterlin BL, Fulton L, Neely ET, Kurihara C, Gupta A, Mali J, et al. Randomized, double-blind, comparative-effectiveness study comparing pulsed radiofrequency to steroid injections for occipital neuralgia or migraine with occipital nerve tenderness. Pain. 2015;156(12):2585-2594.

30. Nagda JV, Davis CW, Bajwa ZH, Simopoulos TT. Retrospective review of the efficacy and safety of repeated pulsed and continuous radiofrequency lesioning of the dorsal root ganglion/segmental nerve for lumbar radicular pain. Pain Physician. 2011;14(4):371-376.

31. Husted DS, Orton D, Schofferman J, Kine G. Effectiveness of repeated radiofrequency neurotomy for cervical facet joint pain. J Spinal Disord Tech. 2008;21(6):406408.

32. Schofferman J, Kine G. Effectiveness of repeated radiofrequency neurotomy for lumbar facet pain. Spine (Phila Pa 1976). 2004;29(21):2471-2473.

33. Kvarstein G. Pulsed radiofrequency - time for a clinical pause and more science. Scandinavian Journal of Pain. 2012;3:124-126.

34. Tsou HK, Chao SC, Wang CJ, Chen HT, Shen CC, Lee HT, Tsuei YS. Percutaneous pulsed radiofrequency applied to the L-2 dorsal root ganglion for treatment of chronic low-back pain: 3-year experience. J Neurosurg Spine. 2010;12(2):190-196.

35. Bogduk N, Fraifeld EM. Proof or consequences: who shall pay for the evidence in pain medicine? Pain Med. 2010;11(1):1-2.

36. Haanpaa M, Attal N, Backonja M, Baron R, Bennett M, Bouhassira D, Cruccu G, et al. NeuPSIG guidelines on neuropathic pain assessment. Pain. 2011;152(1):14-27.

37. Graven-Nielsen T, Arendt-Nielsen L. Assessment of mechanisms in localized and widespread musculoskeletal pain. Nat Rev Rheumatol. 2010;6(10):599-606.

38. Kaptchuk TJ, Stason WB, Davis RB, Legedza AR, Schnyer RN, Kerr CE, Stone DA, et al. Sham device v inert pill: randomised controlled trial of two placebo treatments. BMJ. 2006;332(7538):391-397.

39. Lindsetmo RO, Stulberg J. Chronic abdominal wall pain - a diagnostic challenge for the surgeon. Am J Surg. 2009;198(1):129-134. 